\title{
LA CALIDAD DE LA FORMACIÓN INICIAL DOCENTE EN CHILE: LA PERSPECTIVA DE LOS PROFESORES PRINCIPIANTES
}

\section{El problema y preguntas para una investigación}

Evaluar la calidad de la formación inicial docente (FID) requiere de un abordaje complejo para el que se han venido desarrollando diferentes estrategias a nivel internacional, orientadas tanto a la retroalimentación de procesos formativos como a provocar la discusión acerca de la legitimidad y efectividad de la formación docente (Darling-Hammond, 2006).

Entre estas estrategias de análisis, Manzi, Lacerna, Meckes y Ramos (2011) señalan al menos cinco, dentro de las que es posible ubicar algunas que se implementan en Chile:

- Evaluación del desempeño de futuros profesores durante su formación.

- Evaluación de desempeño en la práctica profesional -como es el caso de la evaluación docente-. ${ }^{3}$

- La evaluación de sus empleadores.

- El análisis de la inserción y retención laboral.

- El uso de pruebas estandarizadas -como es el caso de las pruebas

3 Evaluación obligatoria a los docentes que se desempeñan en el sistema municipal, con el objetivo de fortalecer la profesión docente y contribuir a mejorar la calidad de la educación (docentemas.cl). 
TEDS-M- ${ }^{4}$ a nivel internacional, o de la prueba INICIA $^{5}$ a nivel nacional.

Dentro de esta última estrategia, se debe incluir la de las pruebas que miden aprendizajes en los estudiantes, como PISA ${ }^{6}$ y TIMSS $^{7}$, a nivel internacional; así como también la prueba SIMCE $^{8}$ en Chile, evaluación que muy incipientemente comienza a utilizarse para medir el aporte de los docentes al aprendizaje de sus estudiantes.

En este escenario evaluativo, la peculiar situación de desregulación del sistema de educación superior chileno -que se ha expresado en autonomía plena de las entidades, en la proliferación de una oferta formativa de bajo costo y amplio nicho como es la Pedagogía y que se ha desarrollado a la par de una disminución en la selectividad de sus postulantes (Cox, Meckes y Bascopé, 2011; Bellei y Valenzuela, 2010)-, con un sistema de acreditación de la oferta formativa en revisión a partir de un diagnóstico que revela considerables debilidades para establecer comparaciones, debido a su frágil nivel de rigurosidad y

4 TEDS-M: Teacher Education Study in Mathematics, de la International Association for the Evaluation of Educational Achievement, IEA. Estudio comparativo internacional respecto de la formación inicial docente en matemáticas para educación primaria y secundaria. Participaron 17 países: Estados Unidos, Alemania, Noruega, Polonia, la Federación Rusa, España, Suiza, Taiwán, Singapur, Tailandia, Malasia, Botswana, Filipinas, Chile, Georgia y Omán, aunque este último no fue contabilizado en los resultados. En Chile, se aplicó en una muestra representativa de 1.400 estudiantes de último año de Pedagogía en Educación Básica (iea.nl/:id=20).

5 El programa evaluativo INICIA constituye una actividad expresamente orientada a objetivar las competencias docentes que permitan retroalimentar procesos y estrategias formativas individuales e institucionales para mejorar la calidad de la formación inicial docente. Se aplica a estudiantes de último año de Pedagogía, a fin de medir el efecto de la formación en sus conocimientos. Actualmente es una evaluación de carácter voluntaria y su eventual obligatoriedad se encuentra en discusión parlamentaria (evaluacioninicia.cl).

6 PISA: Programme International of Student Assessment de la OCDE, que evalúa conocimientos aplicados y destrezas en matemáticas, ciencias naturales y lectura para estudiantes de 15 años.

7 TIMSS: Estudio Internacional de Tendencias en Matemática y Ciencias de la Asociación Internacional para la Evaluación del Rendimiento Educativo (IEA). Evalúa los resultados de la implementación de elementos comunes del currículo de matemáticas y ciencias naturales en $4^{\circ}$ y $8^{\circ}$ año, donde Chile ocupa en matemáticas para $4^{\circ}$ básico el lugar 37 entre 50 países y el lugar 37 entre 42 países en matemáticas de $8^{\circ}$ básico; lugar 34 entre 50 países en ciencias de $4^{\circ}$ básico, y lugar 25 entre 42 países en ciencias en $8^{\circ}$ básico.

8 SIMCE se crea el año 1988 para mejorar la calidad y equidad educativa, y desde 2012 es el sistema de evaluación que la Agencia de Calidad de la Educación utiliza para medir los resultados de aprendizaje de los establecimientos. Se aplica censalmente a los estudiantes del sistema escolar en distintos momentos de su escolaridad obligatoria, a fin de evaluar el logro de los contenidos y habilidades del currículo vigente (simce.cl). 
a la falta de procedimientos estandarizados para la toma de decisiones (Domínguez y Meckes, 2011), sumado a evidentes conflictos de interés en el proceso, debido a que las instituciones son clientes de las agencias acreditadoras (Cox, et al., 2011). Todo ello constituye un contexto que ha generado en los últimos años la implementación de algunos sistemas de control, como la acreditación obligatoria de las carreras de Pedagogía y la ya señalada prueba INICIA, además de algunos dispositivos aplicados solo en el sistema municipal, como el programa AEP (Asignación de Excelencia Pedagógica) ${ }^{9}$, también voluntario, al que pueden postular los docentes a fin de obtener reconocimiento al mérito pedagógico y la antes mencionada evaluación docente, todas ellas iniciativas diseñadas para mejorar la calidad de la educación, poniendo a prueba la eficacia de la oferta formativa docente.

Desde la perspectiva de los resultados en las pruebas nacionales e internacionales que miden logros de aprendizaje de los estudiantes, se observa que en la versión más reciente de la prueba PISA (año 2009) Chile se ubicó en el lugar 44 entre 65 países en lectura, esto es bajo el promedio de la OCDE. Una situación similar se aprecia en los resultados de la última prueba TIMSS (año 2011), siendo Chile uno de los países con mayor incremento en sus logros, pero ubicándose siempre bajo el promedio OCDE.

En tanto, en la evaluación internacional TEDS-M -que evalúa la formación inicial docente en matemáticas para educación primaria y secundaria-Chile se ubicó en el lugar 15 entre 16 países participantes (Tatto, Schwille, Ingvarson, Rowley y Peck, 2012). Los resultados de las evaluaciones nacionales de profesores -como la evaluación docente y la prueba INICIA- también indican que la formación inicial docente en Chile no logra los estándares de calidad que precisa. La evaluación docente de 2012 a profesores que ejercen en el sector municipal indica que el 23\% de los evaluados no alcanza el nivel mínimo de desempeño, cifra que ha disminuido paulatinamente desde 2009, cuando más del $30 \%$ de los evaluados obtuvo una calificación insatisfactoria; en tanto

9 Pueden postular al Programa de Acreditación para la Asignación de Excelencia Pedagógica del Ministerio de Educación los docentes en forma voluntaria, y tiene por objetivo ayudar a fortalecer la calidad de la educación mediante el reconocimiento del mérito profesional (aep.mineduc.cl). 
que la prueba INICIA -que desde 2010 permite una aproximación a distinciones entre desempeños de docentes formados en diversas instituciones-, por ahora muestra heterogeneidad dentro de un escenario básico de precariedad.

Los últimos datos disponibles para INICIA (2012) muestran heterogeneidad en los conocimientos de los futuros profesores. La proporción de estudiantes medidos en esta última versión representa solo el 14\% del universo total de egresados de carreras de Pedagogía, de manera que es necesario ser cautelosos respecto de cuán representativos pueden ser sus resultados. Sin embargo, los datos de mediciones anteriores no resultan significativamente distintos, pese a no ser comparables por tratarse de poblaciones e instrumentos diferentes, puntualmente, no se encontraban alineados en relación con los estándares, a diferencia de la última medición.

En este escenario el panorama no resulta muy auspicioso: el $60 \%$ de los egresados obtuvo la calificación de «insuficiente» en el área de conocimientos disciplinarios, y más de un tercio (34\%) alcanza resultados del mismo tipo en conocimientos pedagógicos, observándose una gran variabilidad por institución: entidades en que el $90 \%$ de sus egresados evaluados logran un nivel insuficiente en conocimientos disciplinarios, versus entidades que alcanzan la misma proporción de egresados evaluados en nivel aceptable o sobresaliente. En cerca del $70 \%$ de las instituciones evaluadas, más de la mitad de sus egresados alcanza resultados insuficientes en conocimientos disciplinarios. En el área de conocimientos pedagógicos la variabilidad es también muy alta: entidades con $86 \%$ de logros de nivel insuficiente, versus entidades sin egresados en dicho nivel de logro; y cerca del $40 \%$ de las instituciones con más de la mitad de sus egresados alcanzando logros de nivel insuficiente.

Un diagnóstico que ofrece evidencias de un escenario que no es homogéneo y acerca del que resulta necesario indagar en las diferencias, debido a que no se dispone de información suficiente y confiable respecto de la calidad de la formación inicial docente que se imparte en la numerosa oferta de las distintas instituciones nacionales de educación superior. 
Los evaluados en esta prueba han sido formados en instituciones y programas de carácter heterogéneo, en el contexto de un escenario altamente segmentado socioeconómicamente que cruza los distintos niveles de nuestro sistema educativo; en tanto que los resultados obtenidos en la prueba INICIA que mide conocimientos disciplinarios están estrechamente asociados a las condiciones de entrada a la carrera, sugiriendo con ello que podrían ser una consecuencia más de las competencias de ingreso de los estudiantes, que de su formación, es decir, una expresión del capital social, cultural y económico previo (evaluacioninicia.cl; Manzi et al., 2011). Sin embargo, se sabe poco de la calidad de la formación recibida.

En el contexto chileno, marcado por una profunda desigualdad y segmentación social y educativa, resulta de interés indagar en el análisis de la calidad de la formación inicial docente y en el nivel de ajuste entre dicha formación y las demandas del ejercicio profesional en distintos contextos, trascendiendo a los resultados de las pruebas estandarizadas en las que las políticas nacionales han venido haciendo énfasis. Este trabajo se propone avanzar en dicha dirección, en particular desde la perspectiva de la subjetividad del actor que se inicia en la docencia, el profesor principiante, y desde la perspectiva de otro actor relevante, su empleador o jefe directo, observando las diferencias según la selectividad de las instituciones formadoras y el contexto de iniciación.

\section{Antecedentes}

Estamos en presencia de los efectos de un conjunto de políticas orientadas al mejoramiento de la formación inicial docente en Chile, como por ejemplo el Programa de Fortalecimiento de la FID, impulsado en la década de 1990 por el Mineduc, en el que un grupo de instituciones implementaron proyectos integrales de reforma autodefinidos; iniciativas a partir de las cuales se incrementaron las experiencias prácticas ofrecidas en los programas de estudio y se asumieron como elemento central del proceso formativo, en respuesta a la necesidad de disminuir la distancia entre la formación y las demandas del sistema escolar (Cox, et al., 2011; Montecinos, Walker, Solís, Núñez, Contreras y Rittershaussen, 2010). Otras políticas de mejoramiento se encuentran, por una parte, en el Programa de 
Mejoramiento de la Educación Superior (Mecesup) -implementado entre 2004 y 2010-, que financió, por vía concursable, proyectos destinados a la especialización disciplinaria en la formación de profesores de segundo ciclo básico; y por otra, en la exigencia de acreditación obligatoria de las carreras de Pedagogía desde 2006.

Considerando estos antecedentes es que el estudio que da origen a estas páginas se orientó a analizar las dificultades que experimentan los profesores principiantes según la selectividad de las instituciones formadoras y el contexto de iniciación, en tanto sustrato de expresión del (des)ajuste formativo. Una sección de la indagación tuvo por objeto aproximarse a la evaluación que los profesores principiantes hacen de la formación inicial recibida. Las preguntas centrales de dicha sección del estudio fueron:

- ¿Cómo evalúan los docentes principiantes de educación básica su formación pedagógica, disciplinar y didáctica?

- ¿Cómo se distribuye esta evaluación según los tipos de instituciones formadoras y el contexto de iniciación?

\section{Algunos elementos conceptuales acerca de los criterios para evaluar la calidad de la formación inicial docente}

La literatura especializada ha descrito algunos factores vinculados con la calidad de la formación inicial docente, como: a) la selectividad del programa formativo; b) la acreditación del programa, y c) la autopercepción de preparación para enseñar de los propios egresados (Manzi et al., 2011).

Ingvarson, Schwille, Rowley, Tatto, Senk y Peck (2011) señalan que la robustez de un sistema de aseguramiento de la calidad de la formación docente estriba en la combinación de tres elementos fundamentales:

- Las regulaciones sobre el ingreso: selectividad de los programas.

- Las regulaciones sobre los procesos formativos: un sistema de acreditación de los mismos velando por ofrecer las mejores oportunidades de aprendizaje. 
- Las regulaciones sobre el egreso: un sistema de habilitación docente que verifique el logro de estándares mínimos de desempeño.

Aplicando este modelo al análisis de los sistemas de formación docente de diferentes países los autores concluyen que los sistemas más selectivos de los postulantes a las carreras pedagógicas, que cuentan con sistemas de acreditación independientes, rigurosos y confiables y con mecanismos de habilitación profesional también independientes, obtienen mejores resultados en las pruebas de conocimientos disciplinarios y pedagógicos de sus egresados.

Bajo este esquema, Chile se viene ubicando entre los países con sistemas de aseguramiento de la calidad de la formación inicial docente más débiles, aunque en los últimos años la situación ha tendido a remontar a partir de la implementación de un conjunto de políticas de mejoramiento como el incentivo estatal a la postulación a carreras de Pedagogía con mejores puntajes en la prueba de selección universitaria, la exigencia de acreditación obligatoria de las carreras de Pedagogía y la prueba INICIA.

Sin embargo, respecto de la selectividad de los programas de formación, los estudios que analizan su impacto muestran hallazgos inconsistentes, razón que no permitiría concluir que las instituciones de alto nivel de selectividad alcancen necesariamente los mejores resultados (Chingos y Peterson, 2010). Particularmente en Chile, debido a la estrecha relación entre la selectividad, los resultados académicos y los factores socioeconómicos, la exclusividad del programa de formación constituye un sesgo socioeconómico y cultural que quizás esté incidiendo en los resultados de la medición de conocimientos disciplinarios al egreso.

En el caso de la acreditación del programa de formación, se ha relevado la importancia de contar con sistemas de aseguramiento de la calidad de los mismos, en particular en países con una heterogeneidad de instituciones y propuestas formativas (Vaillant, 2007). Sin embargo, existen escasos datos empíricos respecto del impacto de estos sistemas en los resultados de la formación. En Chile, pese a que la gran mayoría de las carreras de Pedagogía se encuentran acreditadas, 
solo una proporción muy marginal de ellas alcanza seis o siete años (máximo periodo de acreditación): el grueso obtiene no más allá de cuatro (cnachile.cl).

Domínguez, Bascopé, Meckes y San Martín (2012) analizaron la correlación entre el número de años de acreditación de las carreras de Pedagogía Básica y el nivel de sus egresados en cuanto a conocimientos disciplinarios y pedagógicos alcanzados en la prueba INICIA, encontrando inconsistencias, hecho que no permite afirmar que una mayor cantidad de años de acreditación se asocie a mejores resultados INICIA. Los autores interpretan la inconsistencia en, al menos, tres perspectivas:

- el foco de la acreditación, no necesariamente ubicado en el logro de aprendizajes,

- la dificultad para asumir confiable y comparativamente los procesos de acreditación ejecutados por las distintas agencias y

- la poca capacidad de discriminación entre instituciones a partir de la escala de calificaciones utilizada ( 1 a 7 años).

Con ello los autores aluden, fundamentalmente, al error en que se incurre al considerar estos indicadores (años de acreditación o resultados INICIA) como evidencias integrales de calidad.

Respecto de las regulaciones al egreso, Chile representa una de las excepciones en el mundo, ya que mide directamente los conocimientos de los futuros docentes (Prueba INICIA), asumidos como elementos constitutivos de sus competencias docentes. Cuando esto ocurre, los resultados suelen usarse como un indicador de la preparación individual para enseñar. Esta modalidad de uso es bastante aislada todavía en el caso chileno y se han utilizado fundamentalmente los resultados agregados, a modo de indicador importante del nivel de conocimientos de los docentes chilenos recién egresados y, solo desde 2010, como un indicador de conocimientos que da cuenta de los resultados de los egresados según la institución formadora (Manzi et al., 2011). Los resultados de esta prueba vienen indicando que «la mayoría de los egresados de Pedagogía Básica no tiene los conocimientos que se requieren para un buen desempeño profesional» (evaluacioninicia.cl). 
Finalmente, el factor relacionado con la autopercepción de los egresados acerca de su propia formación, en términos de la relación entre dicha percepción y sus propios resultados, es descrito como aún menos claro en la literatura. Específicamente en el caso chileno, en el estudio internacional de TEDS-M, los egresados encuestados manifiestan una muy positiva autopercepción respecto de su preparación en comparación con la que se observa entre egresados de otros países. Paradojalmente, tal como se señalara anteriormente, Chile se ubica en los lugares más bajos del ranking internacional en esta evaluación (Ávalos y Matus, 2010).

Manzi et al. (2011) analizaron la percepción acerca del dominio de distintos ámbitos propios de su formación de los egresados de Pedagogía Básica en 2010 y que rindieron la prueba INICIA, a partir de los resultados del cuestionario complementario de dicha prueba. De esta forma encontraron que se percibían mejor preparados en conocimientos disciplinarios de lenguaje alcanzando el 10\%, lo que implica debilidad en tal sentido, mientras el 21\% se reportaba débilmente preparado en conocimientos disciplinarios de matemáticas. Respecto de la preparación en conocimientos disciplinarios de ciencias sociales y naturales la percepción de debilidad se ubicó en torno al 15\%.

Respecto de los conocimientos en didáctica de estas disciplinas, la percepción de mayor debilidad en la preparación se encontró en la didáctica de las ciencias sociales y naturales, que se ubicó en torno al $16 \%$, seguida de $13 \%$ en matemáticas y ubicándose la menor percepción de inseguridad en la didáctica del lenguaje, con solo 9\% de egresados que perciben debilidad en ella. Una todavía menor percepción de inseguridad se observa en pedagogía y didáctica general, con solo 6\% que se declara débilmente preparado.

También detectaron que la mayoría de los egresados se percibe bien preparado en la planificación de clases (89\%), la evaluación de aprendizajes (85\%) y el manejo de grupo (86\%). Y en considerable menor medida se perciben bien preparados para enseñar a estudiantes con necesidades educativas especiales: solo el 47\%. Estas autopercepciones se reportan de manera agregada, para el grupo total de egresados encuestados. 
Este documento profundiza de forma particular en este factor de autopercepción y, precisamente, en la evaluación que los egresados y sus jefes directos hacen de la formación de los primeros, en términos de una aproximación más detallada y, por cierto, subjetiva, a la calidad del proceso formativo y sus distintas dimensiones, especialmente considerando que la idea del desajuste entre la formación inicial y las demandas del sistema escolar es señalada recurrentemente por expertos internacionales -desde el Informe de la OCDE de 2004- y también por expertos nacionales -en 2005 por la Comisión sobre FID, en 2006 por el Consejo Asesor Presidencial y en 2010 por el Panel de Expertos para una Educación de Calidad-. Se plantea entonces una inquietud ante las limitadas conexiones existentes entre el proceso formativo, el desarrollo profesional y las necesidades de los establecimientos educacionales (Latorre, 2006).

En la misma línea, Vaillant (2004) describe un escenario de omisión del conjunto de las relaciones entre formación inicial docente, ejercicio profesional y condiciones de desempeño de los docentes, que genera profesionales insuficientemente preparados para el ejercicio de su profesión. Flores (2008) pone énfasis en la crítica reiterada hacia la distancia entre la teoría y la práctica como principal nudo de la formación inicial docente, responsable de la falta de preparación de los futuros profesores para enfrentar las actividades cotidianas del quehacer de la enseñanza.

Durante esta fase de iniciación docente, los profesores experimentan el desajuste entre la formación y las demandas a su desempeño, objetivándose en lo que Veenman (1984) acuñó en el concepto «choque de realidad», dando cuenta del impacto que experimentan los profesores principiantes al enfrentarse a situaciones distintas de las que conocían a partir de su formación inicial (Veenman, 1984; Ávalos, Carlson y Aylwin, 2005).

Respecto del impacto del contexto en el desempeño y dificultades de la iniciación docente, Eirín, García y Montero (2009) señalan que el aprendizaje docente depende de la instrucción recibida y del contexto y la cultura en que se desenvuelve el individuo, ya que 
el conocimiento se construye socialmente. En este sentido, considerar el contexto en la mirada de profesores principiantes sobre su propia formación inicial se vuelve muy relevante, en tanto constituye un elemento que puede influir en la aparición y/o grado de determinadas dificultades durante el proceso de iniciación docente, atribuibles a determinadas debilidades formativas.

Desde esta perspectiva no es menor que los contextos de iniciación se encuentren consistentemente asociados, como ya se venía señalando, a la institución de formación inicial (y con ello, al nivel socioeconómico), asumiendo una circularidad en la que los docentes provenientes de entidades menos selectivas tienden a ejercer en establecimientos con las mismas características que sus instituciones de egreso, las que a su vez son establecimientos educativos con las mismas características en que ellos cursaron sus estudios básicos y medios (Ruffinelli y Guerrero, 2009; Meckes y Bascopé, 2010; Cox et al., 2011).

El presente documento intenta avanzar en las diferenciaciones relativas a la evaluación de la formación recibida entre egresados formados en programas con distintos niveles de selectividad y que se insertan en diferentes contextos de iniciación docente.

\section{Metodología}

Se desarrolló un estudio comparativo de naturaleza cuantitativa, sustentado en la aplicación de una encuesta online durante el segundo semestre de 2011, a docentes principiantes (con hasta tres años de experiencia), egresados entre 2008 y 2010 de carreras de Pedagogía Básica de instituciones de educación superior chilenas.

Las encuestas abordaron dimensiones relativas a la caracterización educativo-laboral de los principiantes y sus contextos de iniciación, la evaluación de la formación inicial recibida y las dificultades y fortalezas del desempeño docente. El diseño muestral fue no probabilístico e intencionado, atendiendo a un criterio de factibilidad, en tanto que los análisis estadísticos desarrollados fueron descriptivos e inferenciales, estos últimos, mediante análisis de diferencias de medias. Se construyeron índices para configurar 
las variables relativas a la satisfacción con la formación inicial docente (satisfacción y autopercepción del nivel de preparación para ejercer), el contexto de iniciación (a partir de la identificación de obstaculizadores y facilitadores de la iniciación) y se clasificaron las entidades formadoras según su selectividad ${ }^{10}$.

Los resultados se presentan sobre 890 encuestas respondidas por docentes principiantes egresados de 23 instituciones de educación superior chilenas ${ }^{11}$ y una encuesta online aplicada a 232 de sus empleadores. Los docentes encuestados provienen de cinco instituciones selectivas $(25,1 \%)$, de catorce instituciones de baja selectividad $(48,9 \%)$ y de cuatro instituciones no selectivas $(26,1 \%)$, reproduciendo, en términos generales, la distribución de instituciones y estudiantes en el sistema de educación superior chileno. Los empleadores respondieron por egresados de 16 entidades de formación docente (34,5\% de egresados de instituciones selectivas, $43,1 \%$ de egresados de entidades de baja selectividad y $22,4 \%$ de egresados de instituciones no selectivas).

La opción metodológica responde a criterios de cobertura y amplitud temática, que se maximizan mediante una consulta masiva, asumiendo la eventual deseabilidad que puedan incluir las respuestas.

10 Para esto se utilizó el criterio básico de la clasificación utilizado por Meckes y Bascopé en la que se promediaron puntajes PSU de ingreso a carreras de Educación Básica para tres años, más la aplicación de algunos ponderadores. Si el resultado de este promedio era superior a 550 puntos en cualquiera de los tres años, la carrera se consideraba selectiva. Para este estudio se contemplaron los puntajes PSU para seis años: 2005, 2006, 2007, 2008, 2009 y 2010, los que fueron promediados para un mayor ajuste de los datos, sin incluir ponderadores. Adicionalmente, se diseñó una clasificación con tres tipos de selectividad: selectiva, baja selectividad y no selectiva, a fin de representar mejor la diversidad de la oferta. De este modo, las instituciones que promediaron 550 puntos o más son consideradas selectivas; las que promediaron entre 450 puntos y 549 son consideradas de baja selectividad, y las que promediaron menos de 450 puntos (incluye las instituciones sin requisito PSU) son consideradas no selectivas.

11 Instituto Profesional de Chile, Instituto Profesional de Providencia, Pontificia Universidad Católica de Chile, Universidad Academia de Humanismo Cristiano, Universidad Alberto Hurtado, Universidad Andrés Bello, Universidad Arturo Prat, Universidad Autónoma de Chile, Universidad Católica de Valparaíso, Universidad Católica Silva Henríquez, Universidad Central, Universidad de Ciencias de la Informática, Universidad de Los Andes, Universidad del Bío Bío, Universidad de Los Lagos, Universidad de Tarapacá, Universidad de Las Américas, Universidad del Desarrollo, Universidad del Mar, Universidad del Pacífico, Universidad Diego Portales, Universidad Metropolitana de Ciencias de la Educación y Universidad Santo Tomás. 
Asimismo, el tipo de muestreo no representativo y la encuesta autoaplicada vía web -ya que se trabajó con las encuestas que los egresados estuvieron dispuestos a responder- implica ciertamente la probabilidad de sesgo de autoselección, aunque se estima que ella es menor, debido a la penetración del uso de internet en este segmento de la población. De todos modos, los resultados no resultan generalizables a los programas considerados ni al sistema nacional de formación inicial, aunque se estima que el estudio aporta valiosa información referencial en el tema, considerando el estado incipiente del desarrollo del conocimiento en este ámbito, así como el alto nivel de dificultad que implica levantar la información representativa necesaria.

\section{Principales hallazgos respecto de la evaluación de la formación recibida}

\subsection{La percepción de ajuste entre formación y demandas del ejercicio docente}

La gran mayoría de los encuestados (85\%) se manifiesta satisfecho o muy satisfecho con su formación inicial docente, sin observarse diferencias estadísticamente significativas según selectividad. Consecuentemente, un todavía mayor porcentaje $(93,7 \%)$ se considera bien o muy bien preparado para ejercer la docencia, y los egresados de entidades selectivas se autoperciben significativamente mejor preparados que los egresados de entidades de baja selectividad o no selectivas. 
Gráfico 1. Autopercepción de preparación para ejercer la docencia, según selectividad.

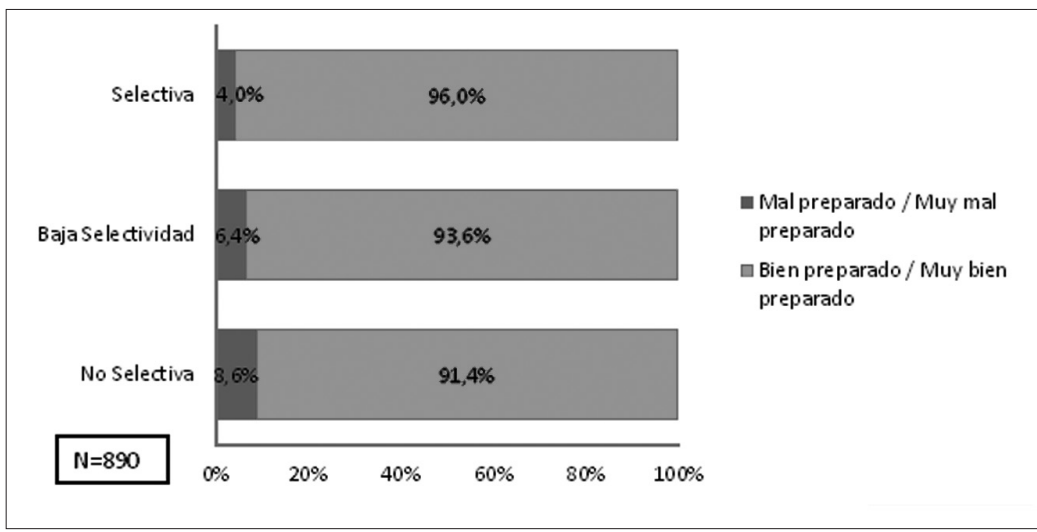

Contrariamente a lo que ha venido señalando la literatura, la percepción de ajuste entre la formación recibida y las demandas reales del ejercicio docente es alta: $72 \%$ de los encuestados estima que dicho nivel de ajuste es alto o muy alto y cerca del $80 \%$ de sus empleadores coinciden con esta apreciación. Esta percepción de ajuste es significativamente mayor entre los egresados de entidades selectivas que entre los egresados de entidades no selectivas o de baja selectividad.

Gráfico 2: En qué medida estimas que la formación inicial que recibiste se ajusta a las demandas reales que tienes para ejercer la docencia, según selectividad

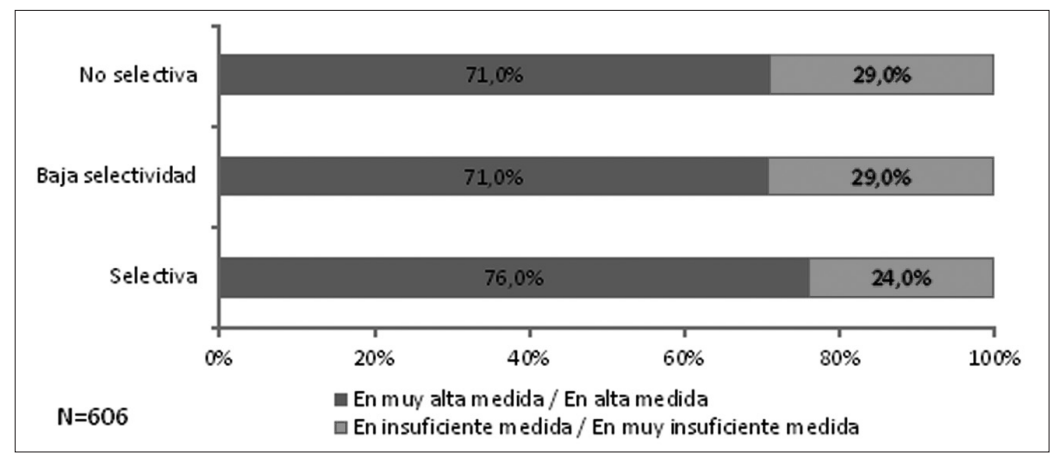

La percepción de ajuste entre formación y demandas del ejercicio docente está mediada por el contexto de iniciación. Entre los docentes que se inician en contextos favorables hay una significativa 
mayor proporción de percepción de ajuste que entre quienes se inician en contextos desfavorables.

Gráfico 3: En qué medida estimas que la formación inicial que recibiste se ajusta a las demandas reales que tienes para ejercer la docencia, según el contexto de iniciación laboral

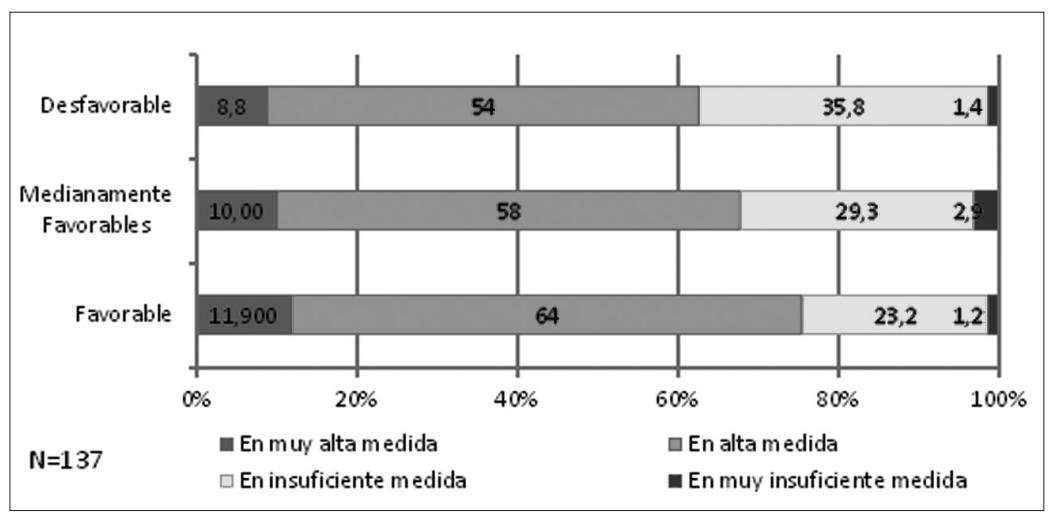

Consistentemente con la evaluación de la formación recibida y su ajuste a las demandas laborales, la autoevaluación del desempeño docente es muy positiva. El 92,6\% de los encuestados estima que se desempeña bien o muy bien, sin observarse diferencias estadísticamente significativas según selectividad. Esta evaluación es muy coincidente con la que hacen sus empleadores o jefes directos: 95\% de ellos se manifiesta satisfecho o muy satisfecho con el desempeño del docente principiante por el que se le consulta, independientemente de la selectividad de la institución formadora.

También la autoevaluación del desempeño está mediada por el contexto de iniciación docente: se observa una significativa mejor autopercepción de desempeño en la medida en que el contexto es más favorable. 
Gráfico 4: Autoevaluación de desempeño docentes principales, según el contexto de iniciación laboral.

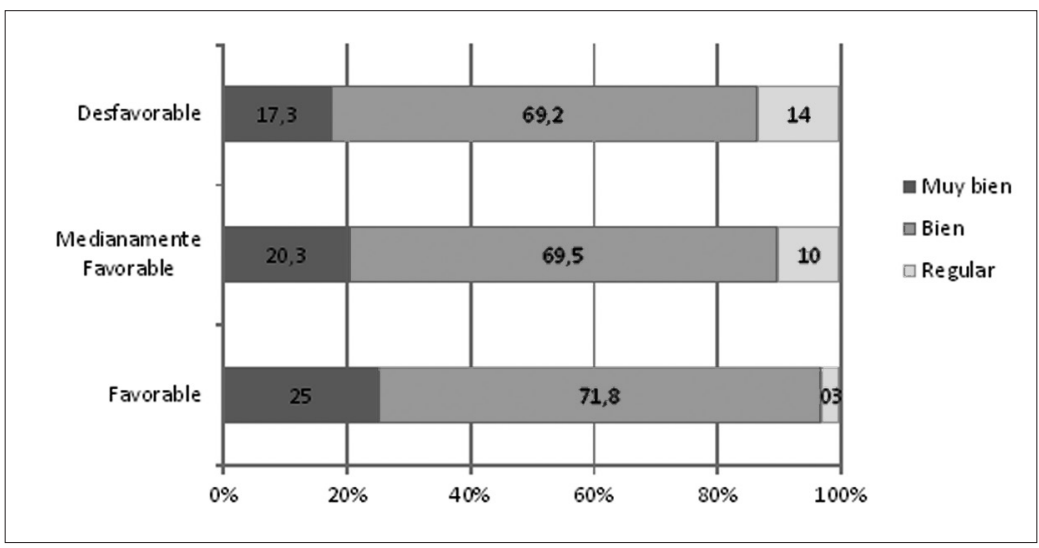

\subsection{La evaluación de la formación}

Los docentes evaluaron ocho áreas de su formación inicial:

- Formación en contenidos disciplinares.

- Formación en el manejo pedagógico de contenidos disciplinares (didáctica).

- Formación pedagógica (currículum, evaluación de aprendizajes, especialización o mención).

- Formación en el manejo de los estudiantes (conocimiento de estudiantes, atención a la diversidad, manejo de la disciplina, motivación y clima en el aula).

- Formación en habilidades interpersonales y trabajo colaborativo.

- Formación en habilidades blandas y adaptación al centro educativo (hábitos laborales: puntualidad, cumplimiento de compromisos, expresión oral y escrita, etc.).

- Formación respecto del sistema educacional chileno.

- Formación práctica.

Un primer aspecto a considerar en la evaluación que los egresados hacen acerca de su formación es que, pese a que el objetivo de los programas es la preparación para enseñar en los ocho primeros niveles de la escuela, la gran mayoría $(81,4 \%)$ se siente mejor preparado para ejercer la docencia en el primer ciclo de la 
educación básica, en tanto que solo el 40,7\% se siente mejor preparado para desempeñarse en segundo ciclo, lo que muestra una debilidad transversal en la formación para el ejercicio en este último ciclo básico, sin observarse diferencias significativas según la selectividad de la entidad de egreso.

Las evaluaciones más deficitarias se concentran en la formación didáctica de ciencias naturales y de ciencias sociales, ya que alrededor de un tercio de los encuestados la considera mala o muy mala, independientemente de la selectividad de la institución formadora. En cuanto a la formación disciplinar de ambas áreas, algo menos de un tercio opina lo mismo. La evaluación de la formación disciplinar en ciencias sociales también resulta ser independiente de la selectividad de la institución formadora. No ocurre igual con la formación disciplinar en ciencias naturales y el egreso de entidades selectivas, donde consistentemente se muestra una evaluación más deficiente: mientras más del 35\% de los egresados de estas entidades estima que dicha formación fue deficitaria, solo alrededor del 26\% de los egresados de entidades de baja y nula selectividad estiman lo mismo.

La mayor concentración de evaluaciones positivas se observa en la formación didáctica y disciplinaria en lenguaje, con 18,5\% y 15\% de los encuestados, respectivamente, que la considera mala o muy mala. Los egresados de entidades no selectivas son más críticos en estas evaluaciones, particularmente respecto de la formación didáctica en lenguaje, con $24,5 \%$ de evaluaciones mala o muy mala, versus $10,4 \%$ de los egresados de entidades selectivas que piensan lo mismo.

El análisis de las diferencias arroja una asociación consistente entre la evaluación deficitaria de la formación tanto en didáctica de las matemáticas como en didáctica del lenguaje y egreso de entidades de baja o nula selectividad: mientras solo el 14,5\% de los egresados de entidades selectivas estima que la didáctica de las matemáticas fue deficitaria, más del 22\% de los egresados de entidades de baja y nula selectividad estima lo mismo; y mientras 10,4\% de los egresados de entidades selectivas estima que la didáctica en lenguaje fue deficitaria, cerca del doble, más del 20\% de los egresados de entidades de baja y nula selectividad opina lo mismo. 
Respecto de la formación disciplinar en matemáticas y lenguaje, la evaluación resulta ser independiente de la selectividad: en torno al 25\% de los encuestados la estima deficitaria en matemáticas; y alrededor del 15\% opina lo mismo en lenguaje.

Gráfico 5: Evaluación de formación disciplinar y didáctica (opción) mala/muy mala) según selectividad.

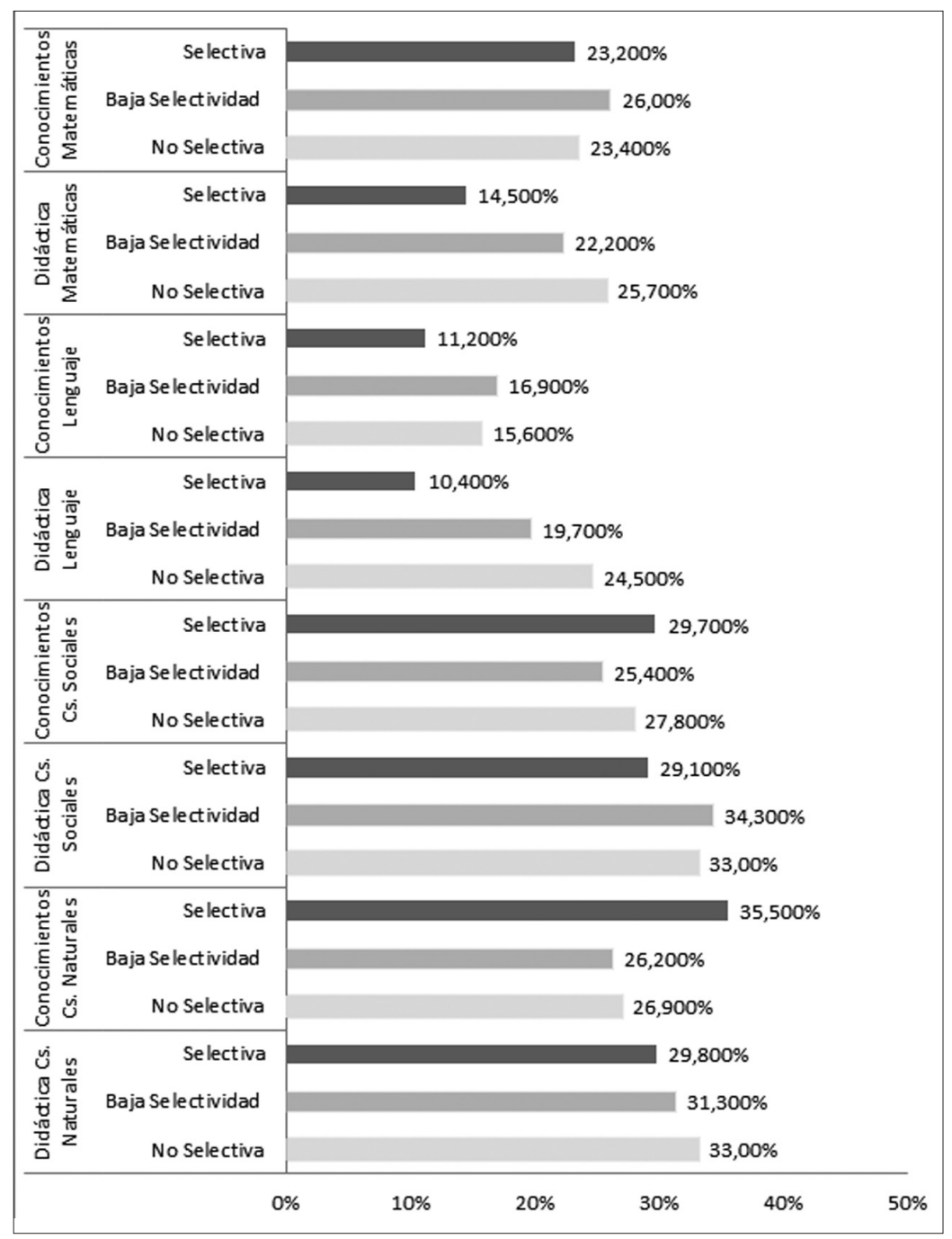

Los docentes principiantes y sus empleadores calificaron el manejo disciplinar y didáctico de los primeros con notas en una 
escala de 1 a 7 . Los resultados son positivos y homogéneos, aunque los profesores principiantes resultan ser más críticos. En general, los profesores se autoevalúan con calificaciones bastante homogéneas, en torno al 5,5, en conocimientos disciplinarios y didácticos. Las mejores autoevaluaciones las hacen en relación con su manejo de conocimientos disciplinarios y didácticos de lenguaje, alcanzando 5,8 promedio en ambos casos, mientras que las evaluaciones más bajas se encuentran en los conocimientos disciplinarios y didácticos de ciencias naturales, con un promedio de 5,3 en ambos casos.

Por su parte, los empleadores califican a los docentes principiantes con notas muy satisfactorias y todavía más homogéneas que la autoevaluación, con alrededor del 6,5 en la mayoría de los ámbitos, evaluando mejor a los principiantes de lo que lo hacen ellos mismos. Las evaluaciones más altas corresponden al manejo de conocimientos disciplinarios y didácticos de las ciencias sociales y naturales, en tanto que las evaluaciones más bajas, aunque siempre muy cercanas a los valores anteriores, corresponden al conocimiento didáctico de lenguaje y matemáticas.

Gráfico 6: Calificación promedio de autoevaluación en manejo disciplinar y didáctico (principiantes, escala 1 a 7 )

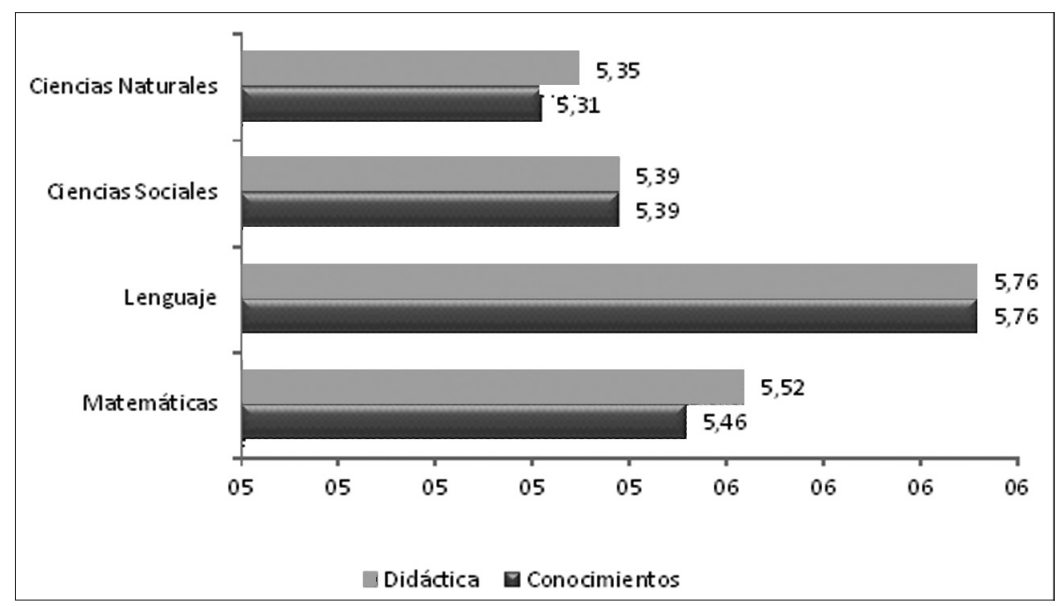


Gráfico 7: Calificación promedio de evaluación en manejo disciplinar y didáctico (empleadores, escala 1 a 7 )

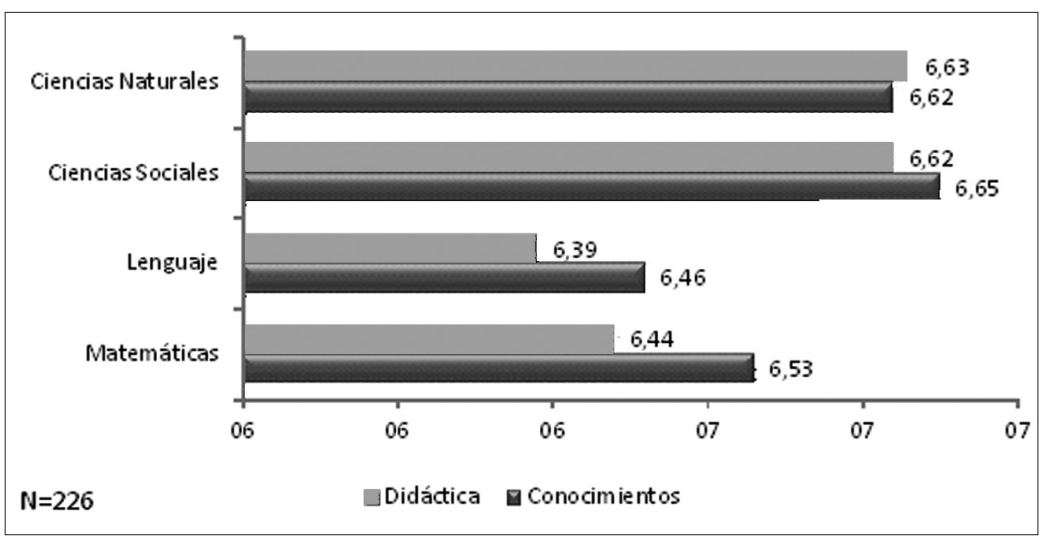

Los aspectos mejor evaluados de la formación inicial se encuentran en la dimensión de formación pedagógica, y fueron: formación pedagógica general, con cerca del 90\% de los encuestados que la evaluó como buena o muy buena, particularmente en currículum, con 87,3\% de los docentes que la evaluó positivamente; seguida de la formación para evaluación de aprendizajes, con 80,4\% que opina de manera similar; sin distinciones según selectividad de la entidad formadora.

El aspecto más débilmente evaluado de esta dimensión fue la formación de especialización o mención, con solo 70,1\% que estima que fue buena o muy buena, calificando significativamente mejor este aspecto de aquellos egresados de entidades selectivas, en comparación con los egresados de entidades no selectivas.

Respecto de la dimensión formación en el manejo de los estudiantes, los aspectos mejor evaluados fueron la formación para la generación de un apropiado clima en el aula: 81,2\% estima que fue buena o muy buena; formación para motivar a los estudiantes, con $80,9 \%$ que la evalúa positivamente; y preparación para conocer a los estudiantes con $80,6 \%$ que la califica como buena o muy buena; sin distinciones según selectividad de la entidad formadora en los dos últimos aspectos, mientras que en la evaluación de la formación para generar un apropiado clima de aula para el aprendizaje son los 
egresados de entidades selectivas significativamente más críticos que sus pares egresados de entidades no selectivas.

Los aspectos más débilmente evaluados de esta dimensión fueron: preparación para atención a la diversidad, donde 71,7\% la evalúa como buena o muy buena; y formación para el manejo del comportamiento en que solo $59,5 \%$ la evalúa como buena o muy buena, correspondiendo al segundo aspecto individual peor evaluado. En ambos aspectos los egresados son significativamente más críticos en la medida en que la selectividad de la institución formadora aumenta.

Llama la atención que la formación recibida para trabajar la diversidad en el aula, ámbito considerado por la literatura como uno de los que concentra las principales dificultades del desempeño, recibe una mejor evaluación que otros aspectos, con menos de un tercio de los encuestados que estima como mala o muy mala la formación recibida en este ámbito.

En la dimensión formación en habilidades interpersonales y trabajo colaborativo se observa el aspecto peor evaluado de todas las dimensiones consultadas: la formación para el trabajo con padres y familias, con $51 \%$ que estima fue mala o muy mala, observándose diferencias significativas según selectividad de la entidad formadora: los egresados de instituciones selectivas son considerablemente más críticos: el 61,5\% estima que la formación recibida en tal sentido fue mala o muy mala, mientras que el $43,4 \%$ estima lo mismo entre egresados de entidades de baja selectividad.

Un aspecto mejor evaluado de esta dimensión -aunque al nivel de los más débilmente evaluados en otras- resulta ser la formación para el trabajo colaborativo con otros profesores, con $71,7 \%$ que considera que fue buena o muy buena, sin diferencias sustantivas según selectividad.

La dimensión formativa relativa a habilidades blandas y adaptación al centro educativo indica que el $81 \%$ estima que fue buena o muy buena respecto de la formación en hábitos laborales (puntualidad, cumplimiento, compromisos, etc.); mientras la 
evaluación es más débil para la formación en expresión oral y escrita, con $72,6 \%$ que considera lo mismo, en ambos casos, sin diferencias significativas según selectividad.

Respecto de la formación en el sistema educacional chileno solo $66,5 \%$ estima que esta fue buena o muy buena, observándose distinciones significativas según selectividad: los egresados de entidades no selectivas evalúan más débilmente este aspecto que los egresados de entidades selectivas. 
Gráfico 8: Evaluación de aspectos de formación, según selectividad

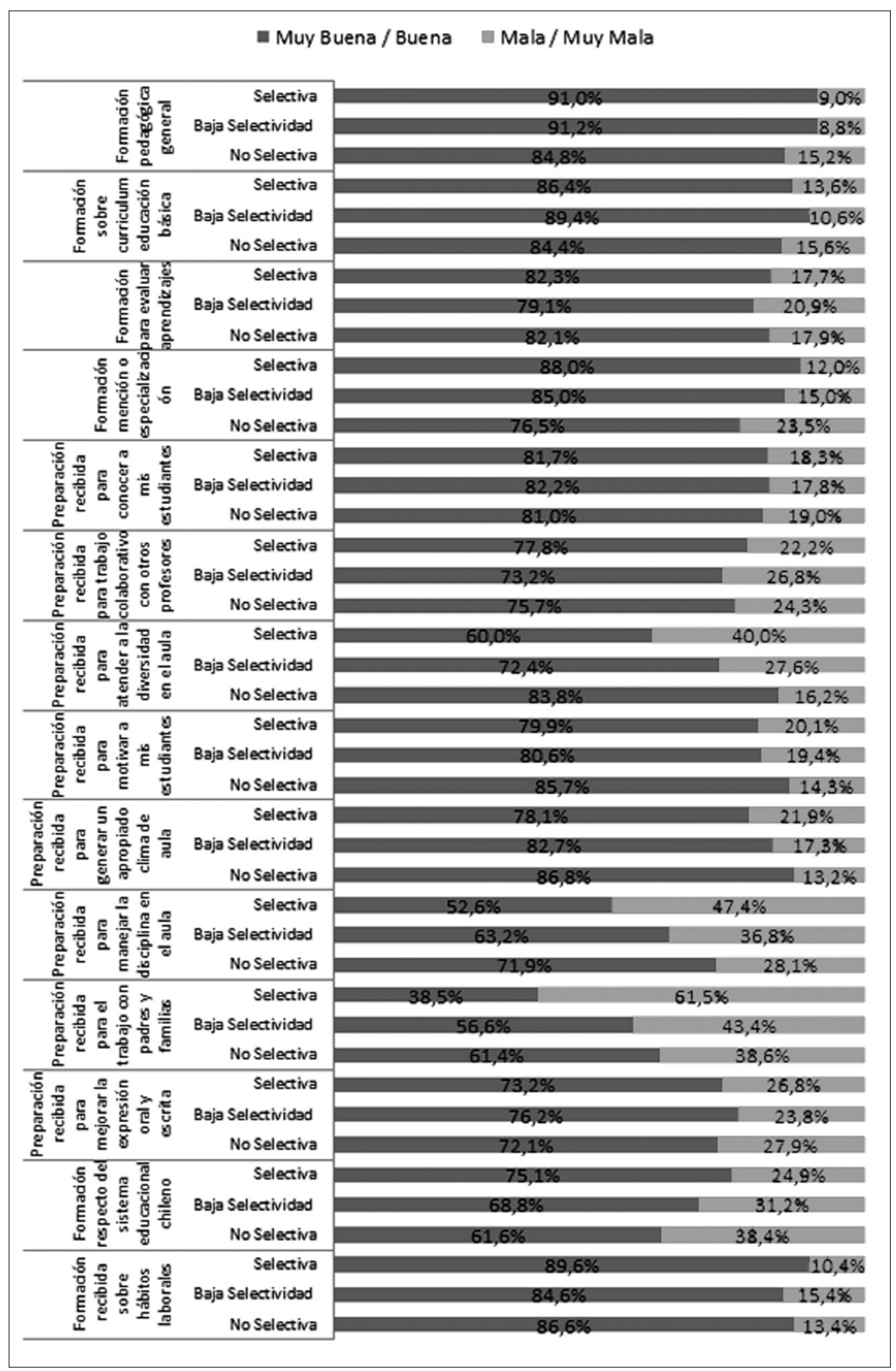

Uno de los aspectos que la literatura consigna como de crucial relevancia para la iniciación docente son las experiencias prácticas en establecimientos educativos durante la formación. El 
91,2\% de los encuestados evaluó como buenas o muy buenas estas experiencias de formación práctica en sus procesos formativos. Las malas evaluaciones se encuentran focalizadas entre los egresados de entidades no selectivas: mientras cerca de 5\% de los docentes egresados de entidades selectivas evalúan mal las prácticas durante su formación, más del triple piensa lo mismo de las suyas entre los egresados de entidades no selectivas, diferencia que resulta ser estadísticamente significativa.

Gráfico 9: Evaluación de las experiencias prácticas en escuelas según selectividad.

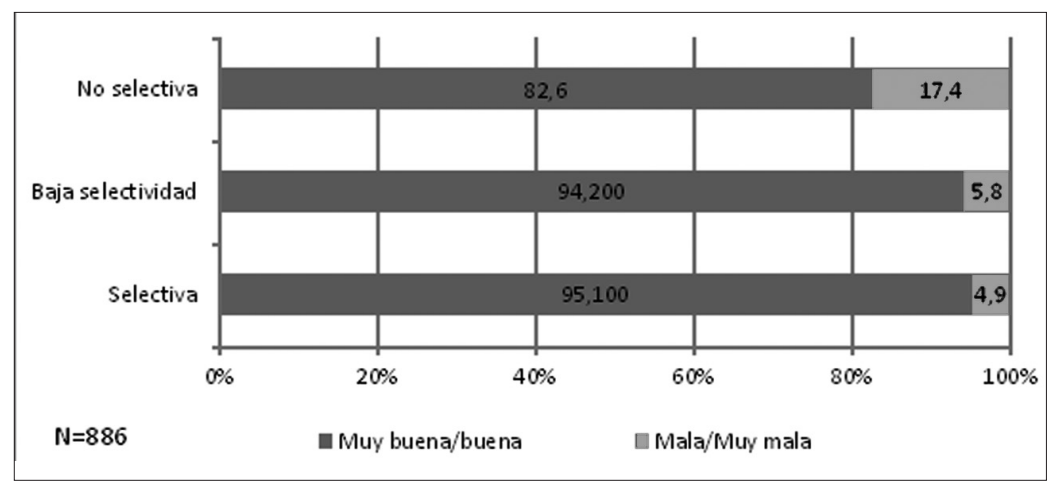

Contrariamente a lo que ha venido indicando la literatura, cerca del $84 \%$ de los encuestados relevó que sus experiencias prácticas resultaron coherentes con lo que habían aprendido en el proceso de formación inicial. Y no se observan diferencias en la evaluación de esta coherencia según selectividad de la institución formadora.

Cuando se profundiza respecto de la supervisión que se hizo a la institución formadora durante las prácticas en las escuelas, la evaluación es también muy positiva: cerca del $81 \%$ de los encuestados señala que fue buena o muy buena. Las malas evaluaciones de supervisión de experiencias prácticas se encuentran nuevamente focalizadas entre los egresados de entidades de baja selectividad y no selectivas. Entre los egresados de estas entidades, las evaluaciones negativas son alrededor del doble que entre egresados de entidades selectivas, y estas diferencias resultan ser estadísticamente significativas. 
Gráfico 10: Evaluación de la supervisión de las prácticas que hacían desde mi institución formadora, según selectividad.

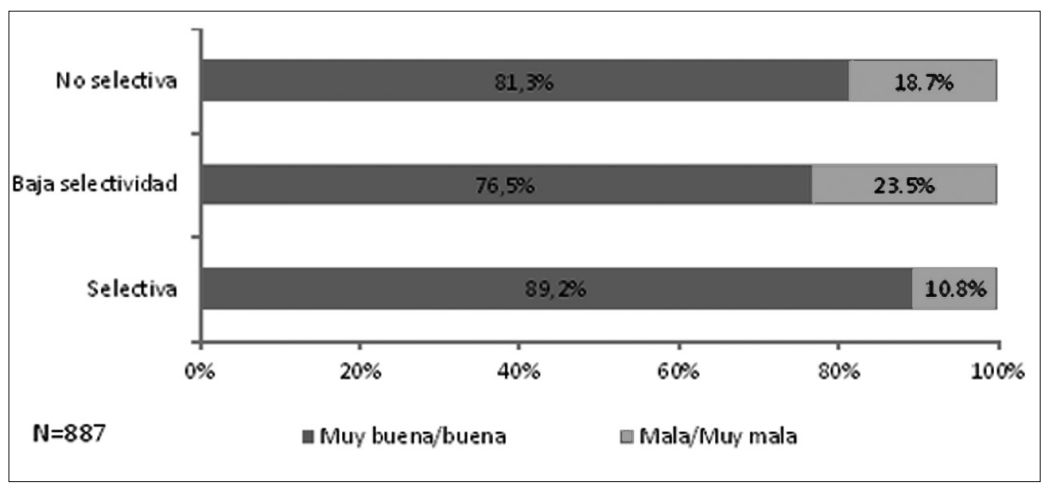

Desde una mirada más global, los principiantes egresados de entidades selectivas estiman en una significativa proporción, mayor que el resto, el haberse formado en su institución, lo que facilitó su iniciación profesional $(77 \%$ de los egresados de entidades selectivas versus el $34 \%$ entre egresados de entidades de baja selectividad y solo $16 \%$ entre egresados de entidades no selectivas).

Gráfico 11: El haber estudiado Pedagogía en la institución y programa en que lo hiciste, hizo que tu iniciación laboral fuera, según selectividad.

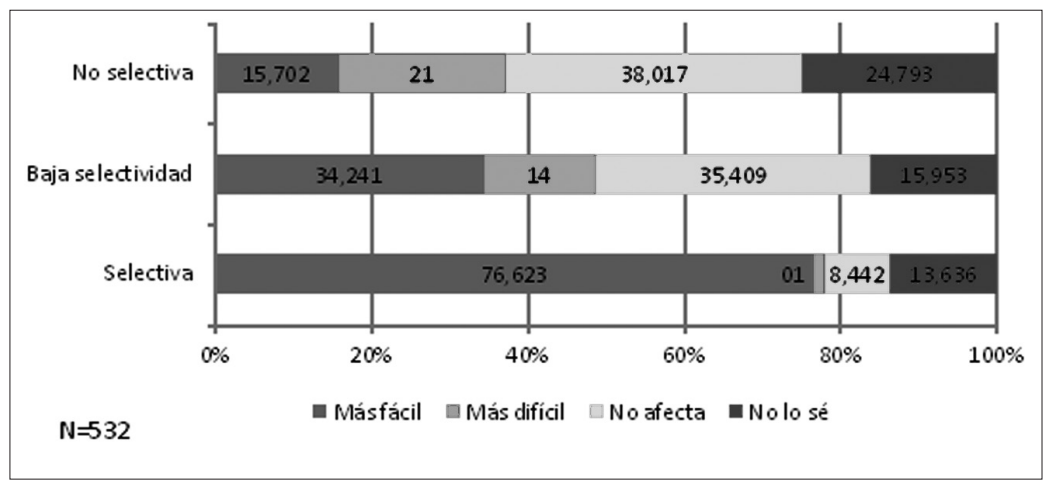

Esta ventaja comparativa no se vincula con apoyos específicos para la inserción, pues los encuestados reportan que solo muy marginalmente recibieron algún tipo de apoyo a su iniciación laboral desde su institución formadora: más del 95\% declara no haber recibido ningún tipo de ayuda y, cuando lo hubo, el apoyo más 
recurrentemente reportado fueron las reuniones para la inserción laboral, pero informadas solamente por 6,1\% de los encuestados.

El análisis de estos apoyos según selectividad indica que se observan diferencias en la presencia de estos dispositivos: en primera instancia a favor de las entidades selectivas, en el caso de la ayuda entregada a los egresados para buscar trabajo y la entrega de otros tipos de apoyo, encontrándose diferencias significativas respecto de los egresados de entidades de baja o nula selectividad y, en segundo término, a favor de las entidades no selectivas respecto de la realización de reuniones o talleres para la inserción laboral de los egresados.

Gráfico 12: Apoyo a la iniciación recibido de la institución formadora, según selectividad.

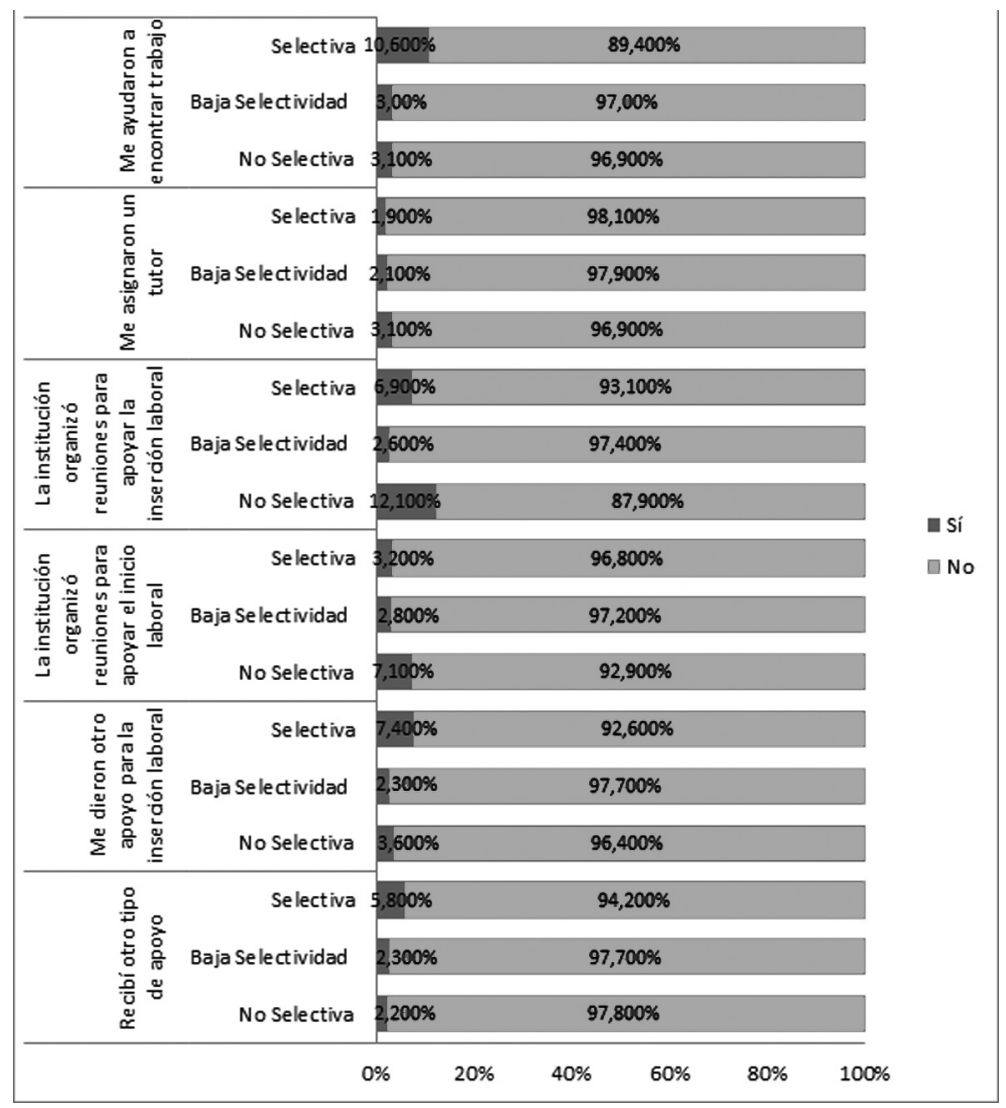


Finalmente, un dato de considerable interés para el análisis es que la gran mayoría de los profesores principiantes encuestados (88\%) manifiesta haber mantenido o fortalecido su decisión de ser profesor tras la experiencia adquirida en su iniciación docente. Solo el 12\% manifiesta que tras la experiencia se ha debilitado su decisión, sin embargo, este fortalecimiento de la decisión es inversamente proporcional a la selectividad de la entidad formadora: entre los egresados de entidades no selectivas y de baja selectividad, tras la iniciación docente, se ha fortalecido la decisión de ser profesor en una proporción significativamente mayor que para los egresados de entidades selectivas.

Gráfico 13: Decisión de ser profesor tras la iniciación docente, según selectividad.

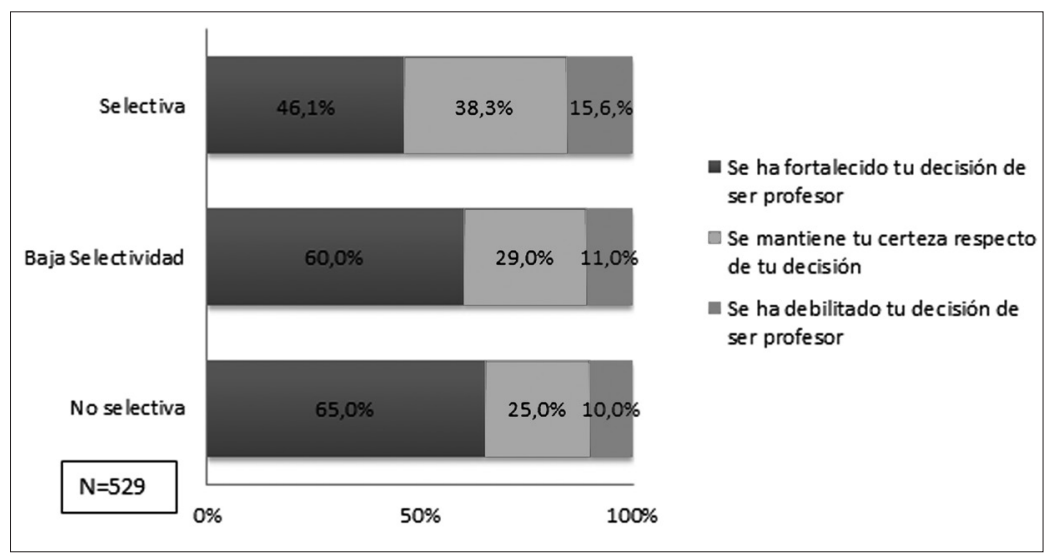

Cerca de un tercio (28\%) de los encuestados ha pensado en la posibilidad de dejar de ejercer la docencia. Y, nuevamente, son los egresados de entidades selectivas quienes lo han considerado significativamente en mayor proporción que el resto del grupo (43\% versus alrededor del 20\%). 
Gráfico 14: Ha pensado en la posibilidad de dejar de ejercer la docencia, según selectividad.

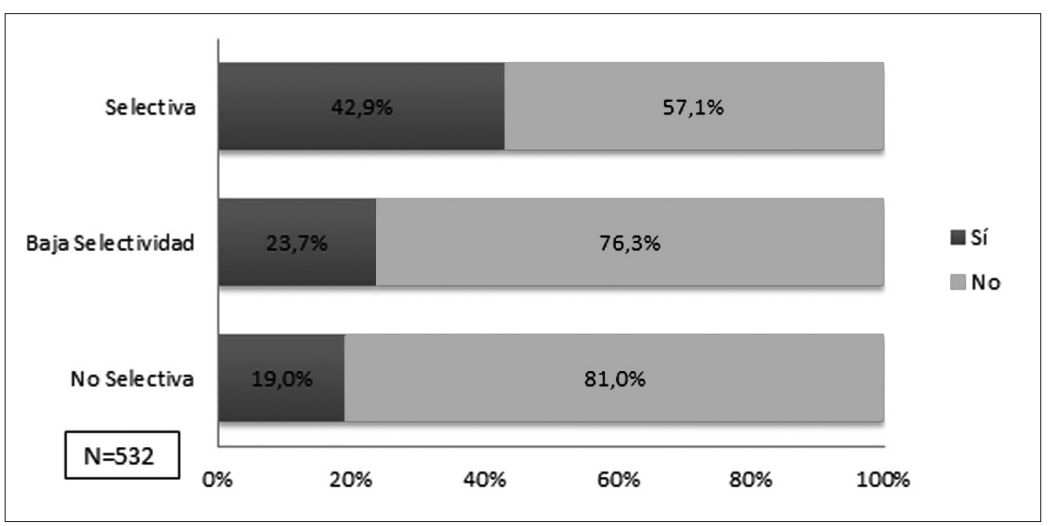

\section{Síntesis y discusión}

En primer término, la evaluación que los propios egresados hacen de su formación ofrece elementos útiles para retroalimentar los procesos formativos, al dar cuenta de fortalezas y debilidades percibidas transversal o diferencialmente en la formación inicial docente, según la selectividad de las instituciones formadoras.

Los hallazgos indican que las fortalezas percibidas en la formación son transversales, encontrándose referencias a una buena evaluación de los siguientes aspectos:

- de la formación pedagógica como currículum y evaluación de aprendizajes;

- de la formación para el manejo de los estudiantes, respecto de la motivación y para su conocimiento;

- de la formación en habilidades blandas y de adaptación al centro educativo.

Se observa que las evaluaciones más positivas de la dimensión formativa disciplinaria y didáctica corresponden a la formación en lenguaje. 
Asimismo, si bien es cierto que la formación disciplinaria y didáctica no destaca como debilidad formativa reportada por los docentes principiantes, los hallazgos dejan en evidencia debilidades transversales de los programas para los siguientes elementos:

- formar sólidamente a sus estudiantes para enseñar en segundo ciclo básico;

- en la formación didáctica en ciencias naturales y sociales;

- en la formación disciplinar en ciencias sociales; y

- en la formación en expresión oral y escrita.

Reviste especial interés detenerse respecto de la formación práctica, dimensión relevada por la literatura por su incidencia crucial en el desempeño e iniciación docente. La gran mayoría valora positivamente este ámbito formativo y las evaluaciones deficitarias se asocian al egreso de entidades no selectivas, y, en una línea discrepante con lo que ha venido indicando la literatura, la gran mayoría estima que sus experiencias prácticas resultaron coherentes con lo que habían aprendido en el proceso de formación inicial, sin distinciones según selectividad.

Evalúan muy positivamente, también, la supervisión en escuelas de las prácticas que se llevó a cabo desde la institución formadora, aunque nuevamente las evaluaciones deficitarias de supervisión se asocian al egreso de entidades de baja selectividad y no selectivas.

Tabla 1: Síntesis de la percepción de fortalezas de la FID según los docentes principiantes

\begin{tabular}{|c|c|c|}
\hline \multicolumn{3}{|c|}{ Fortalezas FID } \\
\hline IES Selectivas & IES Baja selectividad & IES No selectivas \\
\hline \multicolumn{3}{|c|}{ Formación pedagógica: currículum, evaluación } \\
\hline \multicolumn{2}{|c|}{ Formación para el manejo de los estudiantes: para motivarlos, para conocerlos } \\
\hline \multicolumn{2}{|c|}{ Formación en habilidades blandas y de adaptación al centro educativo } \\
\hline
\end{tabular}


Tabla N²: Síntesis de la percepción de debilidades de la FID según los docentes principiantes

\begin{tabular}{|l|l|l|}
\hline \multicolumn{2}{|c|}{ Debilidades FID } \\
\hline \multicolumn{1}{|c|}{ IES Selectivas } & \multicolumn{1}{|c|}{ IES Baja selectividad } & IES No selectivas \\
\hline \multicolumn{2}{|c|}{ Formación para enseñar en 2º́n didáclo básico } \\
\hline \multicolumn{2}{|c|}{ Formación de disciplinar en ciencias naturales yociales sociales } \\
\hline \multicolumn{2}{|c|}{ Formación en expresión oral y escrita } \\
\hline $\begin{array}{l}\text { Formación para el trabajo con padres } \\
\text { y familias }\end{array}$ & $\begin{array}{l}\text { Formación didáctica en matemáticas y lenguaje } \\
\text { Formación práctica } \\
\text { Formación para el manejo de } \\
\text { los estudiantes: Manejo del } \\
\text { comportamiento, de la diversidad y del } \\
\text { clima de aula. }\end{array}$ & $\begin{array}{l}\text { Formación pedagógica: especialización o mención, } \\
\text { formación sobre sistema educativo chileno }\end{array}$ \\
\hline $\begin{array}{l}\text { Formación disciplinar en ciencias } \\
\text { naturales }\end{array}$ & \\
\hline
\end{tabular}

Esta breve síntesis de la percepción general de fortalezas y debilidades de la FID desde la perspectiva de los profesores principiantes parece dar cuenta de logros transversales derivados de más de dos décadas de implementación de programas destinados al mejoramiento de la formación inicial, asociados fundamentalmente a acciones de fortalecimiento de las líneas de prácticas y su vinculación con la dimensión formativa teórica; en la dimensión disciplinar y didáctica de lenguaje y matemáticas y en la dimensión de formación pedagógica.

Del mismo modo, los hallazgos hablan de debilidades transversales que parecen derivar de los mismos esfuerzos de mejoramiento de las últimas décadas, como efectos no deseados, en términos del desmedro que habría significado, por ejemplo, para la formación disciplinar y didáctica en ciencias y otras áreas del conocimiento, la focalización en lenguaje y matemáticas; o el deterioro en la preparación para enseñar en segundo ciclo a partir de una focalización en los aprendizajes básicos del primer ciclo; ello parece hablar de las debilidades del sistema para revertir bajos niveles de competencias generales de los estudiantes a su ingreso a carreras de Pedagogía.

A diferencia de las fortalezas, las debilidades encontradas difieren según la selectividad de la institución formadora, permitiendo identificar elementos constitutivos de esta heterogeneidad formativa. 
Las debilidades asociadas a las instituciones selectivas se concentran en aspectos referidos a:

- la formación para el trabajo con padres y familias,

- la formación para el manejo de sus estudiantes: para el manejo del comportamiento, de la diversidad y del clima de aula; y

- debilidades en la formación disciplinar en ciencias naturales.

Por su parte, la formación en entidades de baja o nula selectividad se asocia a debilidades tanto en los aspectos de la formación pedagógica específica (formación didáctica en matemáticas y lenguaje, formación práctica, formación de especialización o mención) como en la formación respecto del sistema educacional chileno.

El análisis diferenciado de debilidades según la selectividad permite inferir que los efectos de las acciones de fortalecimiento de la FID de estas últimas décadas se observan con mayor claridad entre los egresados de instituciones selectivas, que corresponden precisamente a las que han sido más beneficiadas con estas acciones, concentrando sus dificultades en dimensiones que no han sido foco de las iniciativas de mejoramiento, como la preparación para el trabajo con padres o en la disciplina de ciencias naturales.

En este análisis resulta de particular interés la dificultad específica que reportan para manejar (motivar, controlar y atender la diversidad) a sus estudiantes. Una hipótesis a estudiar podría vincularse con el círculo de segmentación educativa que tiende a destinar a los docentes al mismo tipo de establecimiento educativo del que egresaron, situación que ubicaría mayoritariamente a los profesores egresados de entidades selectivas en establecimientos educativos de también alta selectividad, con estándares también altos de exigencias académicas y conductuales, ante los que se perciben con mejores herramientas para resolver las demandas académicas y no así las conductuales o diferencias individuales.

En cambio, los docentes egresados de entidades de baja o nula selectividad describen las debilidades formativas críticas relativas a dimensiones fundamentales, aludiendo a las didácticas 
básicas, las especializaciones y la formación práctica como las más deficitarias, precisamente los aspectos formativos que las acciones de mejoramiento de las últimas décadas han enfatizado y de las que se han mantenido más al margen estas instituciones. Desde esta perspectiva, se configura una situación desmedrada en la formación impartida por estas entidades, la que podría ser equiparable a una fase evolutiva anterior del resto del sistema.

Finalmente, desde esta perspectiva evaluativa, no resulta menor que la influencia de haberse formado en determinada institución -para efectos de la iniciación profesional- sea percibida de manera significativamente más positiva por egresados de entidades selectivas y que esta ventaja comparativa no se vincule con apoyos específicos para la inserción, que solo son reportados muy marginalmente por egresados de entidades selectivas, cuando se trata de un apoyo directo para encontrar trabajo y por egresados de entidades no selectivas, cuando se trata de ayuda menos directa (como la realización de reuniones o talleres para la inserción profesional de los egresados), confirmándose la ampliación del círculo de la inequidad y la segmentación, desde la formación (y antes de ella) al ejercicio profesional.

Del mismo modo, si bien es cierto que la gran mayoría mantiene o fortalece su decisión de ser profesor tras su iniciación docente, este fortalecimiento resulta ser inversamente proporcional a la selectividad de la entidad formadora, asociándose la posibilidad de dejar de ejercer la docencia al egreso de entidades selectivas. Tampoco resulta menor que esto ocurra, pese a las ventajas comparativas que los egresados de entidades selectivas reconocen en su formación -incluidas las aproximaciones a la vida profesional en las prácticas durante el proceso formativo-, evidenciándose una dificultad del sistema para retener a estos docentes en ejercicio, dificultad que a todas luces trasciende las debilidades del proceso formativo.

Una dimensión de fundamental interés en este sentido (sin considerar las condiciones del ejercicio) se vincula con la idea del continuo formativo y la complejidad que implica implementar dispositivos de apoyo orientados a los profesores que se inician, más 
allá de la definición acerca de la responsabilidad que le compete a la formación o al mercado laboral en esta tarea y trabajar desde una perspectiva colaborativa que permita asegurar que los principiantes reciban el apoyo requerido para completar su formación inicial en el ejercicio e insertarse profesionalmente en forma exitosa.

En segundo término, la transversalidad de la percepción de un alto nivel de satisfacción con la formación inicial recibida entre docentes y sus empleadores (quienes, en su mayoría, son también profesores formados en el mismo sistema), así como la de un alto y transversal nivel de satisfacción con el desempeño docente, dan cuenta de una percepción de ajuste entre la FID y las demandas reales del ejercicio, que podría ser leído como un avance sustantivo en la calidad de la formación inicial, sin embargo, la percepción de ajuste se asocia consistentemente a docentes egresados de instituciones selectivas y también a contextos de iniciación favorables, quienes también se perciben sustancialmente mejor preparados para ejercer que el resto.

En este escenario, resulta fundamental abordar el análisis de la brecha entre los resultados de las evaluaciones a los docentes recién egresados y la autopercepción que tienen de su propia formación y desempeño, generándose dos interpretaciones muy distantes de las debilidades formativas que acusan las evaluaciones docentes que se vienen realizando en el país. Analizar la inconsistencia entre la positiva valoración de la formación y los resultados de aprendizaje que están evidenciando docentes y estudiantes en las mediciones nacionales estandarizadas, como INICIA y SIMCE, hace plausible hipotetizar que algo (no) estaría ocurriendo en los procesos formativos que tendría como resultado entre sus egresados un precario nivel de reflexión acerca del saber y quehacer docente.

Las constataciones relativas a la brecha entre los resultados de las evaluaciones y la autopercepción de los docentes principiantes, así como de las fortalezas y debilidades transversales y diferenciadas de su formación, sugieren la necesidad de nuevas indagaciones que profundicen respecto de si estas debilidades y fortalezas se relacionan más con características efectivas de los procesos formativos, o si más bien se vinculan con el nivel de pensamiento reflexivo de los 
estudiantes de Pedagogía al ingreso a la carrera, asociado a una falta de capacidad de los itinerarios formativos para compensar las debilidades en sus competencias de ingreso.

Si se asume que la construcción de los procesos de mejoramiento de la formación inicial docente debe fundarse en una necesidad que emerge de los propios actores, entonces aparece nítida la necesidad de construir primeramente esas condiciones, a partir de la implementación de estrategias efectivas de análisis y reflexividad crítica en los distintos niveles del sistema formativo, incorporándolos activa, formal y explícitamente a los procesos formativos en Pedagogía.

Del mismo modo, será de interés dilucidar si se observan itinerarios formativos provechosos que efectivamente agregan valor a los estudiantes y los transforma en docentes competentes, así como también lo será el profundizar en los efectos de la experiencia de la iniciación en distintos contextos en torno a los cambios que operan en el desempeño, en la autopercepción del mismo y en la valoración de la formación recibida.

\section{Referencias bibliográficas}

Agencia de la Calidad de la Educación, (2010) «Resultados evaluación PISA 2009, Programa Internacional de evaluación de estudiantes». [En línea], Santiago, disponible en http://www.agenciaeducacion. cl/wp-content/uploads/2013/04/PISA-Programa-Internacional-deEvaluaci\%C3\%B3n-de-Estudiantes.pdf [rescatado el 19 de julio de 2013]

Ávalos, B.; Carlson, B. y P. Aylwin, (2005) «La inserción de profesores neófitos en el sistema educativo: ¿cuánto sienten que saben y cómo perciben su capacidad docente en relación con las tareas de enseñanza asignadas?». Proyecto Fondecyt 1020218. [En línea], Santiago, disponible en: http://www.cepal.org/ddpe/publicaciones/sinsigla/xml/7/19597/ INSERPROFE.pdf_[rescatado el 19 de julio de 2013]

Ávalos, B. y C. Matus, (2010) «La formación inicial docente en Chile desde una perspectiva internacional: Informe Nacional del Estudio Internacional IEA TEDS M». [En línea], Santiago, disponible en: http://issuu.com/ kimun/docs/libro_formaci_n_inicial_docente__iea-teds_m__beat [rescatado el 19 de julio de 2013] 
Bellei, C. y J. Valenzuela, (2010) «¿Están las condiciones para que la docencia sea una profesión de alto estatus en Chile?», presentación del Centro de Investigación Avanzada en Educación, Universidad de Chile para el Coloquio Centro de Politicas Comparadas de Educación. Universidad Diego Portales. Santiago, 28 de octubre de 2010. [En línea] disponible en: http://www.cpce.cl/ponencias-pucon2010/cat_view/38-coloquioscpce [rescatado el 19 de julio de 2013]

Comisión sobre Formación Inicial de Docentes (FID), (2005) «Informe Comisión sobre Formación Inicial Docente». Santiago, Ministerio de Educación y Organización de Estados Iberoamericanos para la Educación, la Ciencia y la Cultura (OEI).

Consejo Asesor Presidencial para la Calidad de la Educación, (2006) «Informe final». 11 de diciembre de 2006, Santiago (en línea). http://www.facso. uchile.cl/psicologia/epe/_documentos/GT_cultura_escolar_politica_ educativa/recursos\%20bibliograficos/articulos\%20relacionados/cons ejoasesorpresidencialparalacalidaddelaeducacion(2006)informefinal. pdf [rescatado el 19 de julio de 2013]

Cox, C.; Meckes, L. y M. Bascopé, (2011) «La institucionalidad formadora de profesores en Chile en la década del 2000: velocidad del mercado y parsimonia de las políticas», en Pensamiento Educativo. $N^{\circ}$ 46-47, pp. 205-245.

Chingos, M. y P. Peterson, (2010) «It's easier to pick a good teacher than to train One: familiar and new results on the correlates of teacher Effectiveness», Economics of Education Review 30 (3), 449-465.

Darling-Hammond, L., (2006) «Assessing Teacher Education: The usefulness of multiple measures for assessing program outcomes», en Journal of Teacher Education. Vol. 57, pp. 120-138.

Domínguez, M. y L. Meckes, (2011) «Análisis y propuestas para la acreditación de Pedagogías en Chile», en Calidad en la Educación. № 34, pp. 165183.

Domínguez, M; M. Bascopé; L. Meckes y E. San Martín, (2012) «¿Producen mejores resultados las carreras de Pedagogía Básica con más años de acreditación?», en Estudios Públicos. № 128, primavera 2012, pp. 1-59.

Eirín, R.; H. García y L. Montero, (2009) «Profesores principiantes e iniciación profesional. Estudio exploratorio», en Profesorado. Revista de currículum y FID del profesorado. Vol. 13, No 1, pp. 101-115.

Flores, M., (2008) «La investigación sobre los primeros años de enseñanza: lecturas e implicaciones», en Carlos Marcelo (coord.) El profesorado principiante. Inserción a la docencia. Barcelona, Octaedro. 
Ingvarson, L.; J. Schwille; G. Rowley; M. Tatto; S. Senk y R. Peck, (2011) National Policies and Regulatory Arrangements for the Mathematics Preparation of Future Teachers in Sixteen Countries. Amsterdam, International Association for the Evaluation of Educational Achievement (IEA).

Latorre, M., (2006) «Nuevas miradas, viejos problemas: las relaciones entre FID inicial y ejercicio profesional», en Foro Educacional. N 10 , pp. 41-63.

Manzi, J.; P. Lacerna; L. Meckes e I. Ramos, (2011) «¿Qué características de la formación inicial de los docentes se asocian a mayores avances en su aprendizaje de conocimientos disciplinarios?», informe final Fonide $N^{\circ}$ F511015. Santiago, Ministerio de Educación.

Meckes, L. y M. Bascopé, (2010) «Distribución inequitativa de los nuevos profesores mejor preparados: características de origen y destino laboral de los egresados de Pedagogía Básica», conferencia dictada durante el Primer Congreso Interdisciplinario de Investigación en Educación. Centro de Investigación Avanzada en Educación, Universidad de Chile y Centro de Estudios de Políticas y Prácticas en Educación, Pontificia Universidad Católica. Santiago, 30 septiembre y $1^{\circ}$ octubre de 2010. [En línea], disponible en: http://www.ciie2010.cl [rescatado el 19 de julio de 2013]

Ministerio de Educación, (2011) «Resultados INICIA 2010». [En línea], Santiago, disponible en: http://www.mineduc.cl/usuarios/cpeip/ doc/201105041721390.INICIA\%202010\%20PPT\%20V2.0.pdf [rescatado el 19 de julio de 2013]

Ministerio de Educación, (2012) «Resultados TIMSS 2011. Estudio internacional de tendencias en matemáticas y ciencias». [En línea], Santiago, disponible en: http://www.mineduc.cl/usuarios/acalidad/ doc/201301151653440.Informe_Resultados_TIMSS_2011_Chile_ (10-01-13).pdf [rescatado el 19 de julio de 2013]

Montecinos, C.; H. Walker; M. Solís; C. Núñez; I. Contreras y S. Rittershaussen, (2010) «Lineamientos para el diseño del currículum del área de formación práctica de las carreras de Pedagogía», en S. Martinic y G. Elacqua (eds.) ¿Fin de ciclo? Cambios en la gobernanza del sistema educativo. Santiago, Facultad de Educación de la Pontificia Universidad Católica de Chile y la Oficina Regional para América Latina de Unesco.

Organización para la Cooperación y el Desarrollo Económicos (OCDE) (2004) «Revisión de políticas nacionales de educación: Chile». [En línea], París, Disponible en: http://www.facso.uchile.cl/psicologia/epe/_ documentos/GT_cultura_escolar_politica_educativa/recursos\%20 
bibliograficos/articulos\%20relacionados/oecd(2004)revisiondepoliti caseducacionenchile.pdf [rescatado el 19 de julio de 2013]

Panel de Expertos para una Educación de Calidad, (2010) «Informe final. Propuestas para fortalecer la profesión docente en el sistema escolar chileno». [En línea], Santiago: Ministerio de Educación. Disponible en: http:// www.mineduc.cl/biblio/documento/201007091211380. Informe\%20final.pdf [rescatado el 19 de julio de 2013]

Ruffinelli, A. y A. Guerrero, (2009) «Círculo de segmentación del sistema educativo chileno: destino laboral de egresados de Pedagogía en Educación Básica», Calidad en la Educación. № 31, 19-44.

Tatto, M.; J. Schwille; L. Ingvarson; G. Rowley y R. Peck, (2012) «Policy, Practice, and Readiness to Teach Primary and Secondary Mathematics in 17 countries: Findings from the IEA Teacher Education and Development Study in Mathematics (TEDS-M)». [En línea] Disponible en: http://works.bepress.com/lawrence_ingvarson1/191 [rescatado el 19 de julio de 2013]

Vaillant, D., (2004) «Construcción de la profesión docente en América Latina. Tendencias, temas y debates». Documento PREAL No 31.

Vaillant, D., (2007) «Mejorando la formación y el desarrollo profesional docente en Latinoamérica», en Pensamiento Educativo. Vol. 41, № 2, pp. 207-222.

Veenman, S., (1984) «Perceived Problems of Beginning Teachers», en Review of Educational Research. Vol 54, № 2, pp. 143-178

\section{Referencias web}

Portal docente más. CPEIP, Ministerio de Educación, Chile. Web: docentemas.cl Portal Evaluación INICIA. Ministerio de Educación, Chile. Web: evaluacioninicia.cl Portal Asignación Excelencia Pedagógica. Ministerio de Educación, Chile. Web: aep.mineduc.cl

Portal Comisión Nacional de Acreditación. Chile. Web: cnachile.cl

Portal de la International Association for the Evaluation of Educational Achievement. Web: iea.nl/?id=20

Portal Agencia de Calidad de la Educación. Ministerio de Educación, Chile. Web: simce.cl

Recibido: 29/07/2013

Aceptado: 25/11/2013 